\title{
Frequent expression of human leukocyte antigen class I and the status of intratumoral immune cells in alveolar soft part sarcoma
}

\author{
AKIRA OGOSE ${ }^{1,2}$, HIROYUKI KAWASHIMA ${ }^{2}$, TETSUO HOTTA ${ }^{2}$, TAKASHI ARIIZUMI ${ }^{2}$, \\ TETSURO YAMAGISHI ${ }^{2}$, NAOKI OIKE ${ }^{2}$, TARO SASAKI ${ }^{3}$, \\ HIROSHI HATANO ${ }^{3}$, HAJIME UMEZU ${ }^{4}$ and NAOTO ENDO ${ }^{2}$ \\ ${ }^{1}$ Department of Orthopedic Surgery, Uonuma Institute of Community Medicine, \\ Niigata University Medical and Dental Hospital, Minami-Uonuma, Niigata 949-7320; \\ ${ }^{2}$ Division of Orthopedic Surgery, Niigata University Graduate School of Medical and Dental Sciences, \\ Niigata 951-8510; ${ }^{3}$ Department of Orthopedic Surgery, Niigata Cancer Center Hospital, Niigata 951-8566; \\ ${ }^{4}$ Division of Pathology, Niigata University Medical and Dental Hospital, Niigata 951-8510, Japan
}

Received November 12, 2015; Accepted December 16, 2016

DOI: $10.3892 / \mathrm{ol} .2017 .5696$

\begin{abstract}
The prognosis of alveolar soft part sarcoma is poor, despite the slow growth of the tumor. A number of cases with spontaneous regression of this rare tumor have been reported. Although the mechanisms underlying spontaneous regression remain uncertain, local immune reaction may be a possible contributing factor. Immunohistochemical expression of human leukocyte antigen (HLA) class I, cluster of differentiation (CD) 3, CD4, CD8, CD20, CD45, CD56, CD68, CD138 and CD163 were assessed in a series of 10 alveolar soft part sarcomas, and the expression profiles were associated with patients' clinicopathological parameters. Expression of HLA class I was observed in almost all the tumor cells of all cases. CD8(+) cells were identified in all tumors with varying densities. Moderate infiltration of CD8(+) cells was detected in three patients; one of these patients survived with long-term tumor remission. Infiltration of CD10(+), CD20(+), CD56(+) or CD138(+) cells was not revealed in all tumors. Moderate-diffuse infiltration of CD163(+) cells was observed in all tumors. To the best of our knowledge, the present study represents the first report of intratumoral immune cells in alveolar soft part sarcoma. Frequent expression of HLA class I in tumor cells was observed. CD8(+) cells were identified at various densities and CD163(+) cells were observed in alveolar soft part
\end{abstract}

Correspondence to: Dr Akira Ogose, Department of Orthopedic Surgery, Uonuma Institute of Community Medicine, Niigata University Medical and Dental Hospital, Urasa 4132, Minami-Uonuma, Niigata 949-7320, Japan

E-mail:aogose@med.niigata-u.ac.jp

Key words: alveolar soft part sarcoma, human leukocyte antigen class I, immune cells, cluster of differentiation 8, cluster of differentiation 163 sarcoma. Moderate infiltration of CD8(+) cells in patients with a good prognosis may indicate the antitumor effects of immune cells in alveolar soft part sarcoma.

\section{Introduction}

Alveolar soft part sarcoma is a morphologically and clinically distinct neoplasm, that was first termed and defined by Christopherson et al (1), in 1952. The name 'alveolar' was derived from its pseudo alveolar appearance, with clustered polygonal cells with eosinophilic cytoplasm (1). Although the tissue origin of alveolar soft part sarcoma still remains unclear, molecular studies have revealed chromosomal rearrangements at translocation $(\mathrm{X} ; 17)(\mathrm{p} 11 ; \mathrm{q} 25)$, resulting in an alveolar soft part sarcoma critical region 1 (ASPSCRI) and transcription factor E3 (TFE3) gene fusion (2-4). The alveolar soft part sarcoma tumor usually presents as a painless, slow growing mass without functional impairment (1). Typically, there is a lack of symptoms, and it is easily overlooked, with metastasis to the lung or brain often the first signs of manifestation of the disease (5-12). Prognosis is poor despite the slow growth of the tumor (5-12). There has been partial initial success with tyrosine kinase inhibitors (13-15), however, active systemic therapy has not been successful for the majority of patients with metastatic diseases $(9,15,16)$. This emphasizes the requirement for alternative treatments such as immunotherapy.

Cases with spontaneous regression of alveolar soft part sarcoma have previously been reported (17-19). However, the mechanisms underlying the spontaneous regression of this type of tumor remain uncertain, and the local immune reaction is considered to be a possible causative factor (20).

Previous studies have demonstrated efficacy of immunotherapeutic strategies against cancer, where antitumor cytotoxic $\mathrm{T}$ lymphocytes are induced by cancer vaccination (21-24). Antitumor responses are modulated by the recognition of immunogenic epitopes in the context of human leukocyte antigen (HLA) class I in the tumors (21). Therefore, 
it is important to evaluate the infiltrations of immune cells and the presence of HLA class I in the tumors. Using immunohistochemistry, the present study evaluated the expression profiles of HLA class I and the presence of intratumoral immune cells in alveolar soft-part sarcoma.

\section{Materials and methods}

Patients. The present study was approved by the Institutional Review Boards of the Niigata University Hospital (Niigata, Japan) and Niigata Cancer Hospital (Niigata, Japan), and complies with the Declaration of Helsinki. Written informed consent was obtained from all patients and/or their families prior to enrollment in the present study. Between January, 1985 and December, 2014, 10 patients with alveolar soft part sarcoma were treated at the Niigata University Hospital and Niigata Cancer Hospital. Data from these patients were reviewed retrospectively. The demographic data for the patients are summarized in Table I. The average age at the time of primary treatment was 32 years (range, 12-75 years). The patient group was comprised of six females and four males. The tumor diameter ranged between 30 and $200 \mathrm{~mm}$ (average $103 \mathrm{~mm}$ ).

Immunohistochemistry. The surgically excised tumor samples were routinely fixed in $10 \%$ buffered formalin for 18-20 $\mathrm{h}$ at room temperature, prior to being embedded in paraffin. A single representative section of each sample ( $4 \mu \mathrm{m}$ thick) was cut and attached to amino silane (APS) coated glass slides (SFRC11; Matsunami Glass Ind., Ltd., Osaka, Japan.). Hematoxylin (Vector Laboratories, Inc. Burlingame, CA, USA) and eosin was used for routine staining. The tissue sections were deparaffinized in xylene and rehydrated in graded ethanol. The slides were steamed for antigen retrieval in $10 \mathrm{mM}$ citrate buffer at pH 6.0 (Dako North America, Inc., Carpinteria, CA, USA) at $85-100^{\circ} \mathrm{C}$ for $40 \mathrm{~min}$. Parts of each sample were treated with $0.2 \%$ trypsin solution at $37^{\circ} \mathrm{C}$ for $20 \mathrm{~min}$, for antigen retrieval. Following cooling for $15 \mathrm{~min}$, the slides were immersed in methanol with $0.3 \%$ hydrogen peroxide for $20 \mathrm{~min}$ at room temperature, in order to block the endogenous peroxidase activity. Subsequently, the slides were incubated with PBS supplemented with $10 \%$ goat serum (Dako North America, Inc.) for 20 min at room temperature, to reduce the occurrence of nonspecific reactions. The slides were then incubated with the primary antibodies at $4{ }^{\circ} \mathrm{C}$ overnight, followed by incubation with a universal immunoperoxidase polymer, including anti-mouse and anti-rabbit antibodies (Histofine Simple Stain MAX PO [MULTI]; dilution 1:1; cat. no. 424141, Nichirei Biosciences, Inc., Tokyo, Japan) for $30 \mathrm{~min}$ at room temperature. The peroxidase activity was detected with 3'-diaminobenzidine tetrahydrochloride (Nichirei). The slides were counterstained with hematoxylin. The following primary antibodies were used: Anti-CD3 (rabbit monoclonal antibody; clone: SP7; dilution 1:1; cat. no. 713591; Nichirei), anti-CD4 (mouse monoclonal antibody; clone:1F6; dilution 1:1; cat. no. 713181; Nichirei), anti-CD8 (mouse monoclonal antibody; clone: C8/144B; dilution 1:1; cat. no. 713201; Nichirei) anti-CD56 (monoclonal mouse anti-human CD56; clone: 1B6; dilution 1:1; cat. no. 713331; Nichirei), anti-CD20 (mouse monoclonal antibody; clone: L26; dilution 1:1,000; cat. no. SL26; Kyowa Hakko Kirin Co., Ltd., Tokyo, Japan), anti-CD45 (monoclonal mouse anti-human antibody; clone: 2B11; dilution 1:200, cat. no. 2B11; Kyowa Hakko Kirin) anti-CD68 (monoclonal mouse anti-human antibody; clone: KP1; dilution 1:5,000; cat. no. Nr.M0814; Dako North America, Inc.) anti-CD138 (monoclonal mouse anti-human CD138; clone: MI15; dilution 1:50; cat. no. Nr.M722; Dako North America, Inc.), anti-CD163 (monoclonal mouse anti-human antibody; clone: 10D6; dilution 1:500; cat. no. NCL-CD163; Leica Microsystems, Inc., Buffalo Grove, IL, USA) and anti HLA class I (mouse monoclonal antibody; clone: EMR8-5; dilution 1:100; cat. no. AB-46; Hokudo Co., Ltd., Sapporo, Japan).

A total of 10 fields of view from each section were assessed by two researchers by light microscopy, and cells were counted manually. The immunohistochemical results were evaluated by semi-quantitative scoring, according to a previous report (23) as follows: +++ , high density of positive cells in the fields; ++ , moderate density and + , low density.

\section{Result}

Clinical course of the patients. At initial presentation, $7 / 10$ patients had metastatic disease and 3/10 patients had developed metastasis following initial treatment. A thigh tumor with eight metastatic lung-nodules at presentation was identified in patient 10 . This patient underwent wide local excision of the thigh tumor, but chemotherapy was not administered, as the patient's parents did not approve the additional cytotoxic treatment. For one year following excision of the primary tumor, the patient received monthly intravenous infusions containing $1 \times 10^{10}$ ex vivo expanded and interferon (IFN) $\alpha$-treated peripheral blood mononuclear cells at another hospital (21). All but one of the lung nodules slowly diminished in size in the six months following the initial surgery. Only one lung-nodule was detected by computed tomography, 24 months following surgery. The size of the lung tumor was stable, and endoscopic resection of the tumor was carried out nine years subsequent to the initial presentation. The patient was free of metastatic disease two years following the metastasectomy (Fig. 1). In total, during the present study, seven patients succumbed to alveolar soft-part sarcoma, two patients survived with metastatic disease, and one patient survived and was free from cancer.

Immunohistochemistry. As presented in Figs. 2-4 almost all tumor cells of the tissue samples stained with the antibody against HLA class I. The expression of HLA class I in soft tissue tumors was examined and the level of expression differed from case to case (data not presented). Infiltration of CD3(+) $\mathrm{T}$ cells was demonstrated in all tumors. Scattered-moderate infiltration of CD4(+) cells was observed in 8/10 of the tumors. CD8(+) cells were revealed in all tissue samples with varying densities. Moderate infiltration of CD8(+) cells was demonstrated in tissue samples 1, 6 and 10 (Figs. 2-4). Intratumoral infiltration of CD10(+), CD20(+), CD56(+) or CD138(+) cells was not revealed in the tumor samples. Moderate-diffuse infiltration of CD163(+) cells was observed in all tumor samples (Figs. 2-4); these cells were interspersed within nests of tumor cells and the perivascular region. CD68 levels were 
Table I. Clinical characteristics of 10 cases with alveolar soft-part sarcoma.

\begin{tabular}{rcclcccr}
\hline Patient & Age (years) & Gender & \multicolumn{1}{c}{ Site } & $\begin{array}{c}\text { Maximum } \\
\text { diameter }(\mathrm{mm})\end{array}$ & $\begin{array}{c}\text { Metastasis at } \\
\text { presentation }\end{array}$ & Prognosis & $\begin{array}{r}\text { Survival } \\
\text { (months) }\end{array}$ \\
\hline 1 & 28 & M & Leg & 70 & Yes & SOD & 124 \\
2 & 61 & M & Thigh & 80 & Yes & SOD & 120 \\
3 & 21 & F & Buttock & 200 & Yes & SOD & 12 \\
4 & 25 & F & Retroperitoneum & 100 & No & SOD & 66 \\
5 & 75 & F & Foot & 70 & No & SOD & 42 \\
6 & 24 & F & Axilla & 120 & Yes & SOD & 10 \\
7 & 14 & M & Leg & 30 & Yes & AWD & 61 \\
8 & 21 & F & Retroperitoneum & 150 & No & SOD & 19 \\
9 & 36 & F & Thigh & 160 & Yes & AWD & 16 \\
10 & 12 & M & Thigh & 55 & Yes & NED & 138 \\
\hline
\end{tabular}

M, male; F, female; SOD, succumbed to disease; AWD, alive with disease; NED, no evidence of disease.

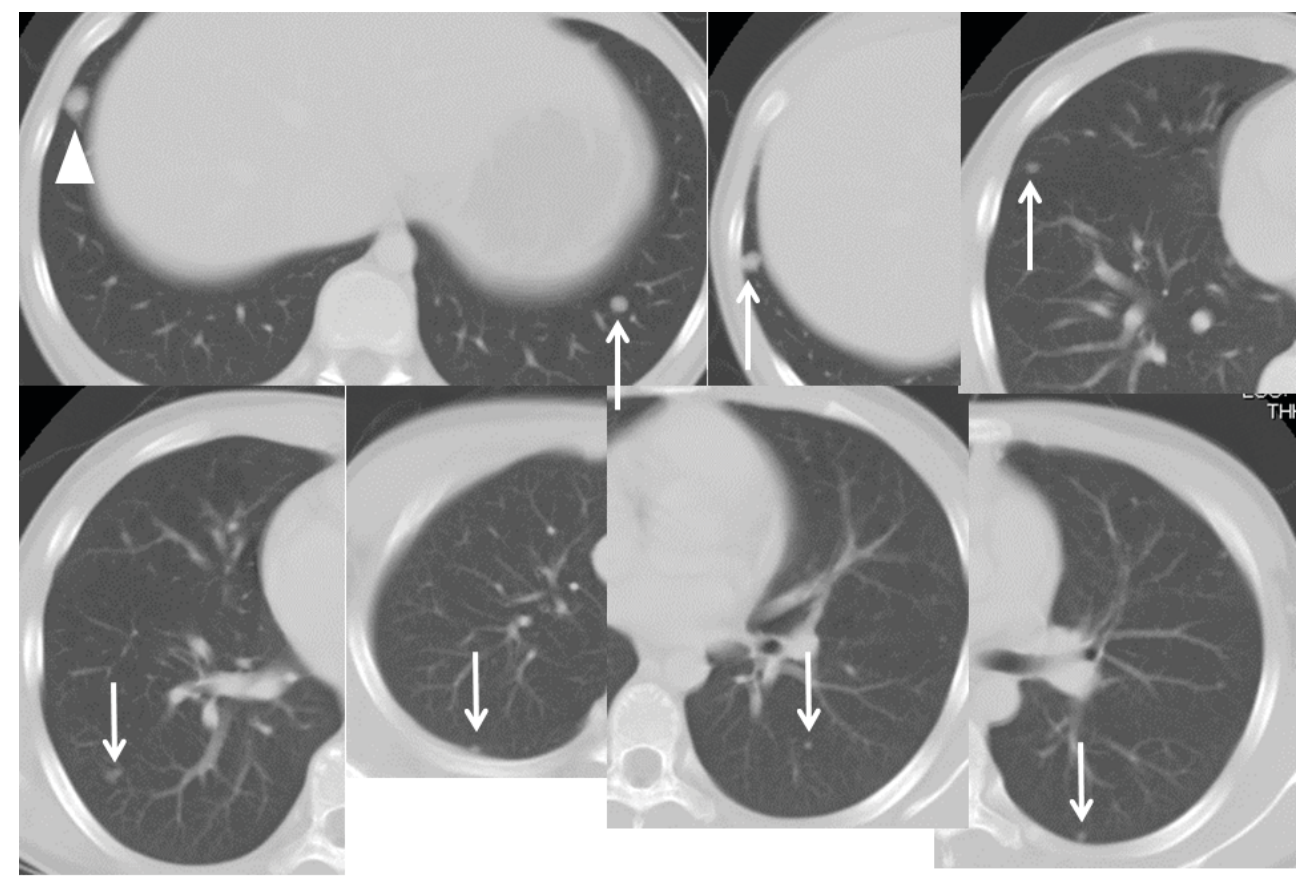

Figure 1. Multiple lung metastases in case 10. Arrows show the regressing nodules following initial the surgery and the arrowhead shows the persistent nodule that was resected seven years later.

analyzed in all tissue samples; however, precise measurements were difficult to obtain as inflammatory cells and numerous tumor cells were positive for this antigen. In case 10 , the metastatic lung tumor cells were also positive for HLA class I, and moderate infiltration of $\mathrm{CD} 8(+)$ cells was revealed in the metastatic tumor tissues (Fig. 5; Table II).

\section{Discussion}

Previous studies have demonstrated five cases of spontaneous regression of alveolar soft part sarcoma (Table III), in which three of the five patients are Japanese $(17-19,25,26)$ The high incidence of Japanese cases may due to language bias during literature review. All three Japanese patients remain alive following an extended period of remission, as determined by verbal communication with the patients. The spontaneous regression of sarcomas is rare and the actual incidence has not been reported (20). Although the underlying mechanism of regression is not fully known, immune mechanisms have been demonstrated in certain types of cancer (20).

The present study revealed moderate infiltration of CD8(+) lymphocytes in patient 10 , who had long-term remission of multiple lung metastases, which suggests an anticancer immune response in this tumor. The specific immunotherapy treatment of this case is not known; however, diffuse infiltration of CD8(+) lymphocytes in the primary tumor prior to immunotherapy suggests specific immune components were activated. CD8(+) cells in malignant tumors have 


\section{A $\operatorname{HE}($ case 2)}

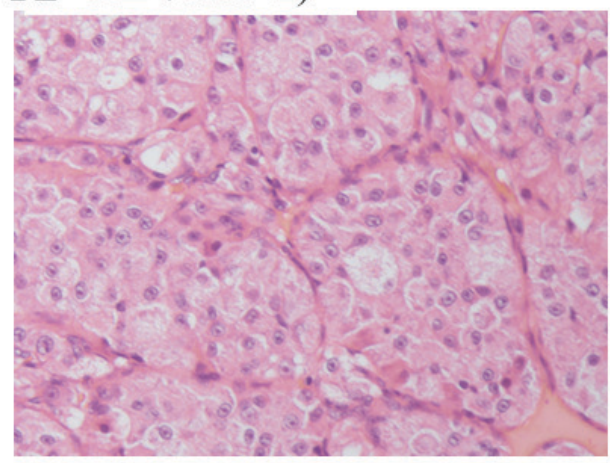

\section{CD8 (case 2)}

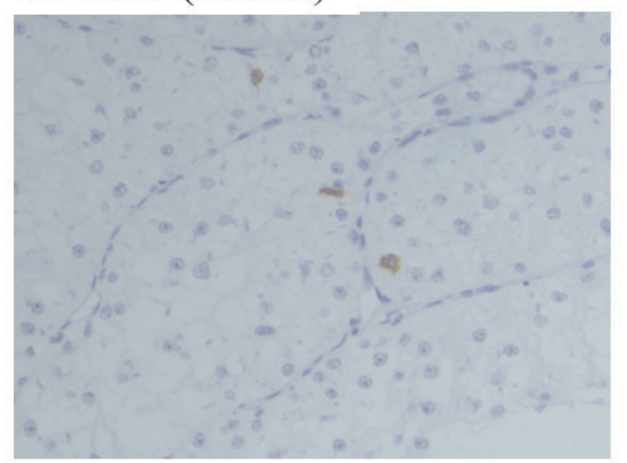

\section{B HLA class I (case 2)}

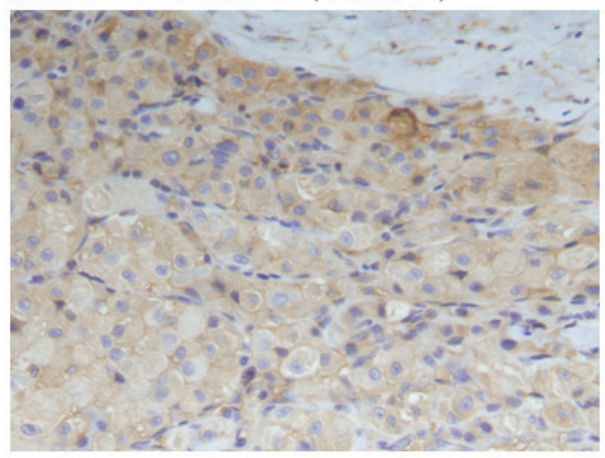

\section{CD163 (case 2)}

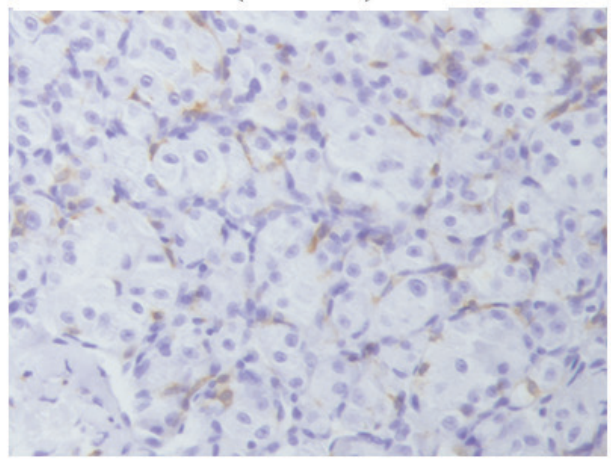

Figure 2. Representative immunohistochemical reactions for HLA class 1, CD8, and CD163 in case 2. (A) HE staining; (B) diffusely positive tumor cells for HLA class 1; (C) scattered infiltration of CD8(+) immune cells; (D) diffuse infiltration of CD163(+) cells. HLA, human leukocyte antigen; CD, cluster of differentiation; HE, hematoxylin and eosin.

\section{A HE (case 6)}

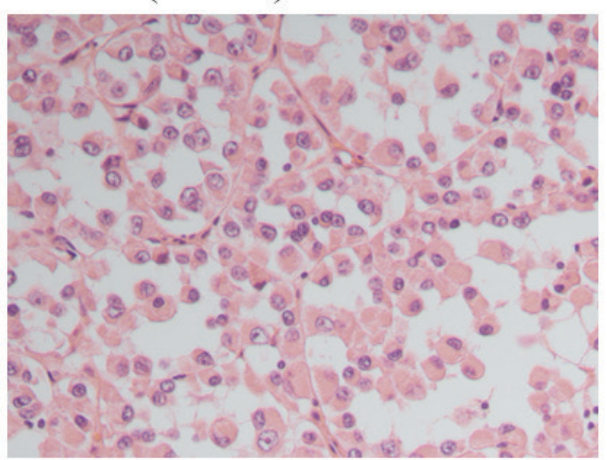

\section{CD8 (case 6)}

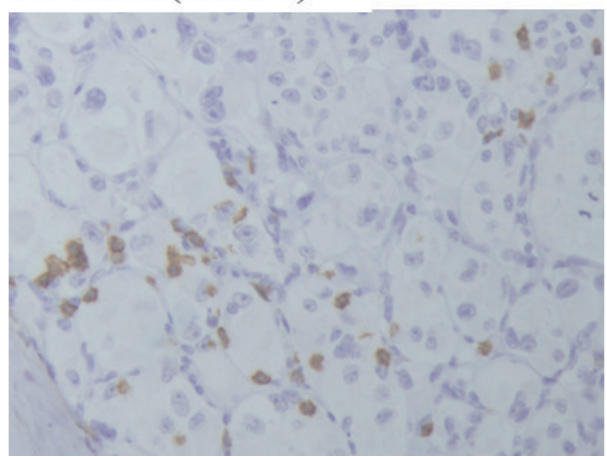

B HLA class I (case 6)

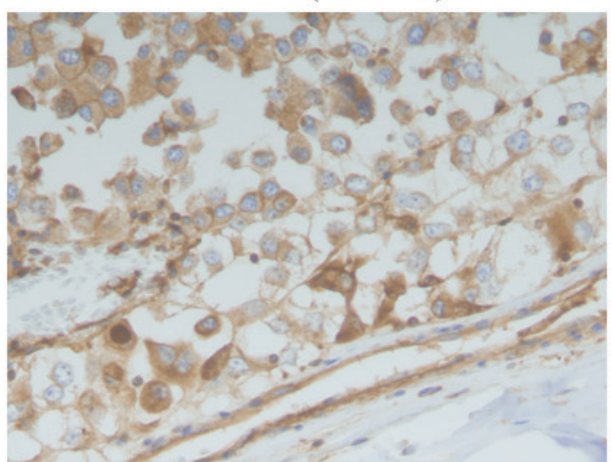

\section{D $\operatorname{CD} 163($ case 6)}

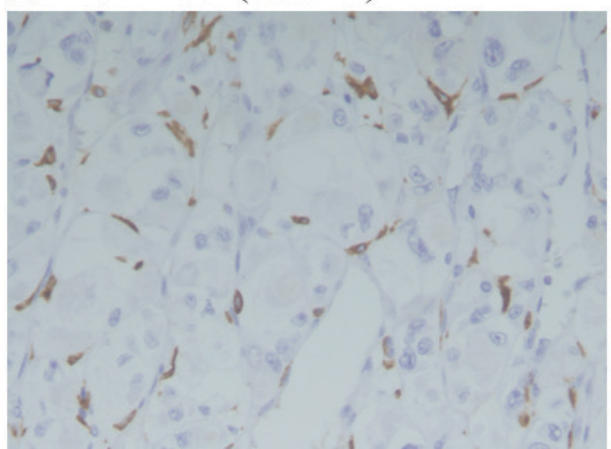

Figure 3. Representative immunohistochemical reactions for HLA class 1, CD8 and CD163 in case 6 (A) HE staining; (B) diffusely positive tumor cells for HLA class 1; (C) moderate infiltration of CD8(+) immune cells; (D) diffuse infiltration of CD163(+) cells. HLA, human leukocyte antigen; CD, cluster of differentiation. HE, hematoxylin and eosin. 
Table II. Results of immunohistochemically analysis of 10 cases of alveolar soft part sarcoma.

\begin{tabular}{|c|c|c|c|c|c|c|c|c|c|c|}
\hline Patient & HLA class I & CD3 & CD4 & CD8 & CD10 & CD20 & CD45 & CD56 & CD138 & CD163 \\
\hline 1 & +++ & ++ & - & ++ & - & - & ++ & - & - & +++ \\
\hline 2 & +++ & + & + & + & - & - & + & - & - & +++ \\
\hline 3 & +++ & + & - & + & - & - & + & - & - & ++ \\
\hline 4 & +++ & + & - & + & - & - & + & - & - & +++ \\
\hline 5 & +++ & + & - & + & - & - & + & - & - & ++ \\
\hline 6 & +++ & ++ & + & ++ & - & - & ++ & - & - & +++ \\
\hline 7 & +++ & + & + & + & - & - & + & - & - & ++ \\
\hline 8 & +++ & + & + & + & - & - & + & - & - & +++ \\
\hline 9 & +++ & + & + & + & - & - & + & - & - & ++ \\
\hline 10 & +++ & ++ & + & ++ & - & - & ++ & - & - & +++ \\
\hline
\end{tabular}

+++, high density; ++, moderate density; +, low density; -, Negative; HLA, human leukocyte antigen; CD, cluster of differentiation.

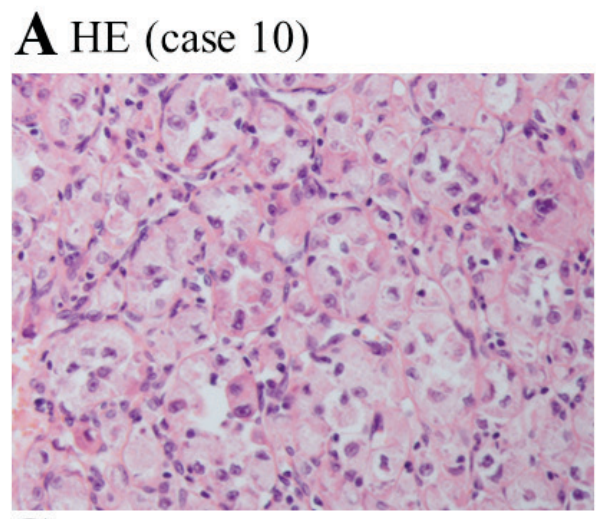

C CD8 (case 10)

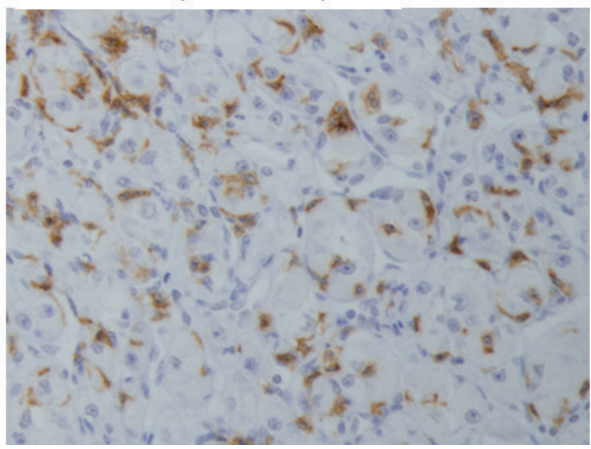

\section{B HLA class I (case 10)}

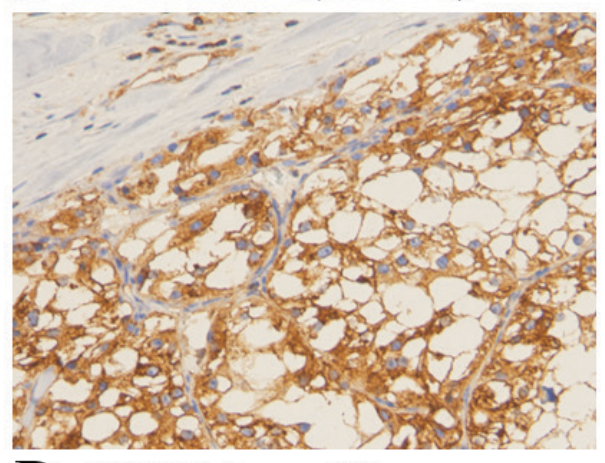

D $\mathrm{CD} 163$ (case 10)

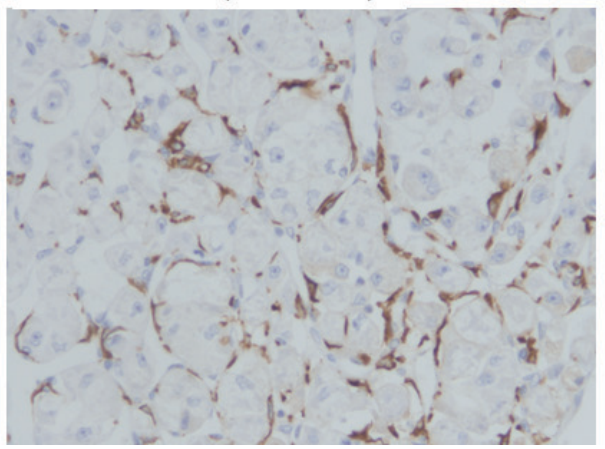

Figure 4. Representative immunohistochemical reactions for HLA class 1, CD8 and CD163 in Case 10. (A) HE; (B) diffusely positive tumor cells for HLA class 1; (C) moderate infiltration of CD8(+) immune cells; (D) diffuse infiltration of CD163(+) cells. HLA, human leukocyte antigen; CD, cluster of differentiation. HE, hematoxylin and eosin.

been associated with an improved survival rate for patients with various types of cancer, including non-small cell lung carcinoma, endometrial carcinoma, melanoma and Ewing sarcoma (20). In the present study, the infiltration of CD10(+), CD20(+), CD56(+) or CD138(+) cells was not identified in all tissue samples. Therefore, B or natural killer cells do not dominate the immune response in alveolar soft-part sarcoma.

The present study was designed to determine the expression levels of HLA class I and infiltration levels of immune cells in alveolar soft part sarcoma. It was revealed that the expression levels of HLA class I were preserved in all cases investigated in the current study. Previously, Tsukahara et al (27) investigated expression levels of HLA class I in bone and soft tissue tumors. Tsukahara et al (27) revealed that HLA class I was not expressed or was downregulated in $63 \%$ of bone and soft tissue tumor samples. Although the sample size in the current study is small, preservation of HLA class I expression and infiltration of CD8(+) lymphocytes in the tumor cells demonstrates noteworthy results in all cases. The results were concordant with those of previous studies, confirming the presence of an immune response in alveolar soft part sarcoma (28). In addition, the diffuse infiltrations of CD8(+) lymphocytes in 


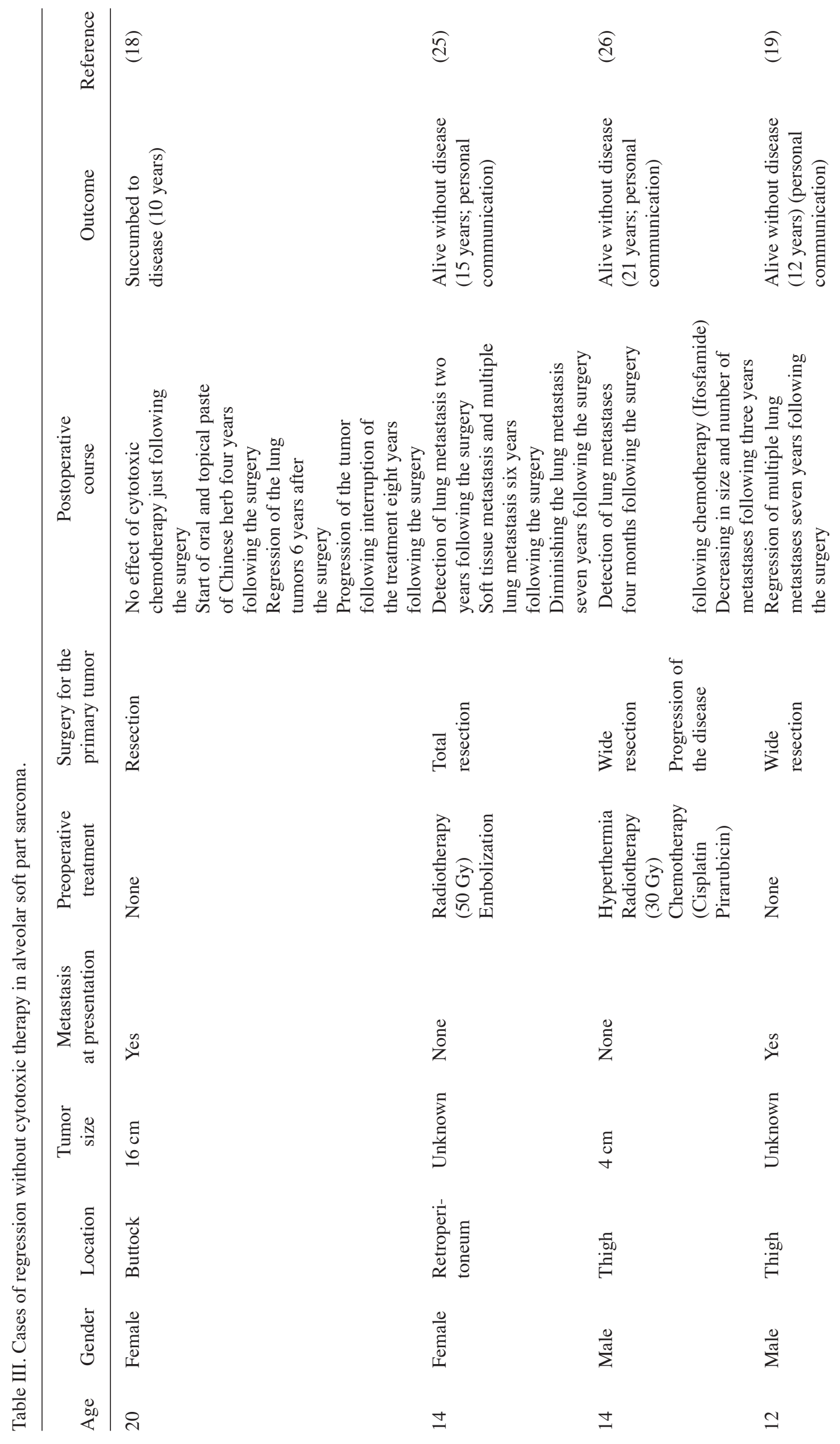


A HLA class1(lung metastasis in case 10)

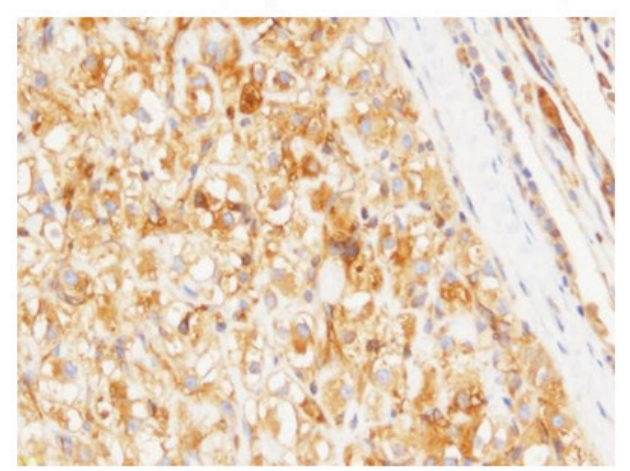

B CD8 (lung metastasis in case 10)

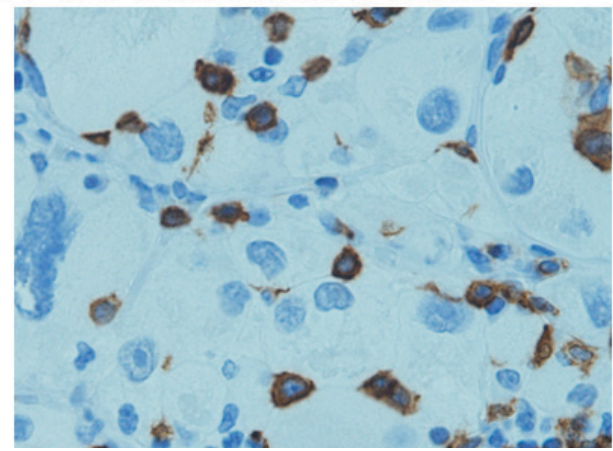

Figure 5. Representative immunohistochemical reactions for HLA class 1 and CD8 (A) diffusely positive tumor cells for HLA class 1; (B) moderate infiltration of CD8(+) immune cells. HLA, human leukocyte antigen; CD, cluster of differentiation. HE, hematoxylin and eosin.

case 10 indicates that the immune response may contribute to improved prognosis of the tumor.

Tumor-associated macrophages are stromal cells that are known to promote tumor invasion, metastasis and angiogenesis in numerous types of cancer (27). The macrophages are able to produce and secrete growth factors, cytokines and other inflammatory mediators that may have important roles in tumor progression (29). These tumor-activating functions are consistent with the results of previous clinical st9dies, demonstrating that the high macrophage density of various types of cancer is associated with a poor prognosis (29). In addition, a previous study also demonstrated that macrophage infiltration is associated with poor prognosis in Ewing sarcoma (30). However, a study of osteosarcoma revealed that macrophages were associated with a good clinical outcome (31). The role of macrophages in sarcoma differs depending on the tumor subtype (31). In the present study, CD163 macrophages infiltrated diffusely in all alveolar soft tissue tumor samples, similar to a previous study (28). The prognostic value of macrophages in alveolar soft part sarcoma remains controversial. Additional studies are required to improve understanding of the role of macrophages in soft tissue tumors.

In conclusion, to the best of our knowledge, the present study represents the first report of intratumoral immune cell presence in alveolar soft part sarcoma. Frequent expression of HLA class I was revealed in the tumor cells. CD8(+) cells were identified at varying densities and CD163(+) cells were present in alveolar soft part sarcoma. Moderate infiltration of CD8(+) cells was observed in the tumors of patients with a 
good prognosis, which may indicate the antitumor effect of immune cells in alveolar soft-part sarcoma.

\section{Acknowledgements}

The authors would like to thank Ms K. Tanaka and Mr Y. Tanaka (Division of Orthopedic Surgery, Niigata University Graduate School of Medical and Dental Sciences, Niigata, Japan) for their technical assistance. The present study was supported in part by a grant from JSPS KAKENHI (grant. no. $15 \mathrm{~K} 10434)$.

\section{References}

1. Christopherson WM, Foote FW Jr and Stewart FW: Alveolar soft-part sarcomas; structurally characteristic tumors of uncertain histogenesis. Cancer 5: 100-111, 1952.

2. Ladanyi M, Lui MY, Antonescu CR, Krause-Boehm A, Meindl A, Argani P, Healey JH, Ueda T, Yoshikawa H, Meloni-Ehrig A, et al: The der (17)t(X;17)(p11;q25) of human alveolar soft part sarcoma fuses the TFE3 transcription factor gene to ASPL, a novel gene at 17q25. Oncogene 20: 48-57, 2001.

3. Isgiguro $\mathrm{N}$ and Yoshida $\mathrm{H}$ : ASPL-TFE3 oncoprotein regulates cell cycle progression and induces cellular senescence by up-regulating p21. Neoplasia 18: 626-635, 2016.

4. Hodge JC, Pearce KE, Wang X, Wiktor AE, Oliveira AM and Greipp PT: Molecular cytogenetic analysis for TFE3 rearrangement in Xp11.2 renal cell carcinoma and alveolar soft part sarcoma: Validation and clinical experience with 75 cases. Mod Pathol 27: 113-127, 2014.

5. Casanova M, Ferrari A, Bisogno G, Cecchetto G, Basso E, De Bernardi B, Indolfi P, Fossati Bellani F and Carli M: Alveolar soft part sarcoma in children and adolescents: A report from the Soft-Tissue Sarcoma Italian Cooperative Group. Annal Oncol 11: 1445-1449, 2000.

6. Hilbert M, Mary P, Larroquet M, Serinet MO, Helfre S, Brisse H, Coulomb A and Orbach D: Alveolar soft part sarcoma in childhood: Is Sunitinib-SutentOे treatment an effective approach? Ped Blood Cancer 58: 475-476, 2012.

7. Kayton ML, Meyers P, Wexler LH, Gerald WL and LaQuaglia MP: Clinical presentation, treatment, and outcome of alveolar soft part sarcoma in children, adolescents, and young adults. J Pediat Surg 41: 187-193, 2006.

8. Lieberman PH, Brennan MF, Kimmel M, Erlandson RA, Garin-Chesa P and Flehinger BY: Alveolar soft-part sarcoma. A clinico-pathologic study of half a century. Cancer 63: 1-13, 1989.

9. Ogose A, Yazawa Y, Ueda T, Hotta T, Kawashima H, Hatano H and Morita T; Japanese Musculoskeletal Oncology Group: Alveolar soft part sarcoma in Japan: Multi-institutional study of 57 patients from the Japanese Musculoskeletal Oncology Group. Oncology 65: 7-13, 2003.

10. Ogura K, Beppu Y, Chuman H, Yoshida A, Yamamoto N, Sumi M, Kawano H and Kawai A: Alveolar soft part sarcoma: A single-center 26-patient case series and review of the literature. Sarcoma 2012: 907179, 2012

11. Pappo AS, Parham DM, Cain A, Luo X, Bowman LC, Furman WL, Rao BN and Pratt CB: Alveolar soft part sarcoma in children and adolescents: Clinical features and outcome of 11 patients. Med Pediatr Oncol 26: 81-84, 1996.

12. Pennacchioli E, Fiore M, Collini P, Radaelli S, Dileo P, Stacchiotti S, Casali PG and Gronchi A: Alveolar soft part sarcoma: Clinical presentation, treatment, and outcome in a series of 33 patients at a single institution. Annal Surg Oncol 17: 3229-3233, 2010.

13. Goldberg JM, Gavcovich T, Saigal G, Goldman JW and Rosen LS: Extended progression-free survival in two patients with alveolar soft part sarcoma exposed to tivantinib. J Clin Oncol 32: e114-e116, 2014.
14. Kummar S, Allen D, Monks A, Polley EC, Hose CD, Ivy SP, Turkbey IB, Lawrence S, Kinders RJ, Choyke P, et al: Cediranib for metastatic alveolar soft part sarcoma. J Clin Oncol 31: 2296-2302, 2013.

15. Mir O, Boudou-Rouquette P, Larousserie F, Blanchet B, Babinet A, Anract P and Goldwasser F: Durable clinical activity of single-agent bevacizumab in a nonagenarian patient with metastatic alveolar soft part sarcoma. Anticancer Drugs 23: 745-748, 2012.

16. Rekhi B, Ingle A, Agarwal M, Puri A, Laskar S and Jambhekar NA: Alveolar soft part sarcoma 'revisited': Clinicopathological review of 47 cases from a tertiary cancer referral centre, including immunohistochemical expression of TFE3 in 22 cases and 21 other tumours. Pathology 44: 11-17, 2012.

17. Banihani MN and Al Manasra AR: Spontaneous regression in alveolar soft part sarcoma: Case report and literature review. World J Surg Oncol 7: 53, 2009.

18. Pang JA, Yeung TF and Cockram CS: Alveolar soft-part sarcoma: A hormone-sensitive tumour? Postgrad Med J 64: 386-388, 1988.

19. Yanagisawa M, Kakizaki $\mathrm{H}$ and Kikuchi A: Alveolar soft part sarcoma: Report of four cases. J Jap Orthopaed Assoc 77 (Suppl): S792-S792, 2003 (In Japanese).

20. Ogihara Y, Takeda K, Yanagawa T and Hirasawa Y: Spontaneous regression of lung metastases from osteosarcoma. Cancer 74: 2798-2803, 1994.

21. Tsukahara T, Emori M, Murata K, Hirano T, Muroi N, Kyono M, Toji S, Watanabe K, Torigoe T, Kochin V, et al: Specific targeting of a naturally presented osteosarcoma antigen, papillomavirus binding factor peptide, using an artificial monoclonal antibody. J Biol Chem 289: 22035-22047, 2014.

22. Tsukahara T, Kawaguchi S, Torigoe T, Takahashi A, Murase M, Kano M, Wada T, Kaya M, Nagoya $S$ and Yamashita T: HLA-A*0201-restricted CTL epitope of a novel osteosarcoma antigen, papillomavirus binding factor. J Transl Med 7: 44, 2009.

23. Yabe H, Tsukahara T, Kawaguchi S, Wada T, Torigoe T, Sato N, Terai C, Aoki M, Hirose S, Morioka H and Yabe H: Prognostic significance of HLA class I expression in Ewing's sarcoma family of tumors. J Surg Oncol 103: 380-385, 2011.

24. Ebina T, Ogama N, Shimanuki H, Kubota T and Isono N: Life-prolonging effect of immunocell BAK (BRM-activated killer) therapy for advanced solid cancer patients: Prognostic significance of serum immunosuppressive acidic protein levels. Cancer Immmunol Immunother 52: 555-560, 2003.

25. Akiyama N, Iida H, Tsuboyama $\mathbf{N}$, Toguchida J and Nakamura T: Alveolar soft part sarcoma with spontaneous regression of lung metastasis: A cse report. Orthop Surg 51: 1295-1297, 2000 (In Japanese).

26. Shiomi I, Otsuka T, Yonezasa M, Kamiyama H, Shibata Y, Tada T and Matsui N: Alveolar soft part sarcoma with spontaneous regression of pulmonary metastases. Clin Orthop Surg 38: 839-841, 2003 (In Japanese).

27. Tsukahara T, Kawaguchi S, Torigoe T, Asanuma H, Nakazawa E, Shimozawa K, Nabeta Y, Kimura S, Kaya M, Nagoya S, et al: Prognostic significance of HLA class I expression in osteosarcoma defined by ant-pan HLA class I monoclonal antibody, EMR8-5. Cancer Sci 97: 1374-1380, 2006.

28. Castelli C, Tazzari M, Negri T, Vergani B, Rivoltini L, Stacchiotti S and Pilotti S: Structured myeloid cells and anti-angiogenic therapy in alveolar soft part sarcoma. J Transl Med 11: 237, 2013.

29. Komohara Y, Jinushi M and Takeya M: Clinical significance of macrophage heterogeneity in human malignant tumors. Cancer Sci 105: 1-8, 2014.

30. Fujiwara T, Fukushi J, Yamamoto S, Matsumoto Y, Setsu N, Oda Y, Yamada H, Okada S, Watari K, Ono M, et al: Macrophage infiltration predicts a poor prognosis for human ewing sarcoma. Am J Pathol 179: 1157-1170, 2011.

31. Buddingh EP, Kuijer ML, Duim RA, Bürger H, Agelopoulos K, Mykebost O, Serra M, Mertens F, Hogendoorn PC and Lankester AC, Cleton-Jansen AM: Tumor-infiltrating macrophages are associated with metastasis suppression in high-grade osteosarcoma: A rationale for treatment with macrophage activated agents. Clin Cancer Res 17: 2110-2119, 2011. 